\title{
Lipidomic analysis of variation in response to simvastatin in the Cholesterol and Pharmacogenetics Study
}

\author{
Rima Kaddurah-Daouk $\cdot$ Rebecca A. Baillie $\cdot$ Hongjie Zhu \\ Zhao-Bang Zeng $\cdot$ Michelle M. Wiest $\cdot$ Uyen Thao Nguyen • \\ Steven M. Watkins $\cdot$ Ronald M. Krauss
}

Received: 18 January 2010/Accepted: 4 March 2010/Published online: 1 April 2010

(c) The Author(s) 2010. This article is published with open access at Springerlink.com

\begin{abstract}
Statins are commonly used for reducing cardiovascular disease risk but therapeutic benefit and reductions in levels of low-density lipoprotein cholesterol (LDL-C) vary among individuals. Other effects, including reductions in C-reactive protein (CRP), also contribute to treatment response. Metabolomics provides powerful tools to map pathways implicated in variation in response to statin treatment. This could lead to mechanistic hypotheses that provide insight into the underlying basis for individual variation in drug response. Using a targeted lipidomics platform, we defined lipid changes in blood samples from the upper and lower tails of the LDL-C response distribution in the Cholesterol and Pharmacogenetics study.
\end{abstract}

Electronic supplementary material The online version of this article (doi:10.1007/s11306-010-0207-x) contains supplementary material, which is available to authorized users.

R. Kaddurah-Daouk ( $₫)$

Duke University Medical Center, Box 3950, Durham,

NC 27710, USA

e-mail: kaddu001@mc.duke.edu

R. A. Baillie

Rosa \& Co. LLC, 751 Laurel St., Suite 127, San Carlos, CA 94070, USA

M. M. Wiest · U. T. Nguyen · S. M. Watkins

Lipomics Technologies-Tethys Bioscience, 3410 Industrial

Boulevard, West Sacramento, CA 95691, USA

H. Zhu $\cdot$ Z.-B. Zeng

Department of Statistics and Bioinformatics Research Center,

North Carolina State University, Raleigh, NC 27695-7566, USA

R. M. Krauss $(\bowtie)$

Children's Hospital Oakland Research Institute, Oakland,

CA 94609, USA

e-mail: rkrauss@chori.org
Metabolic changes in responders are more comprehensive than those seen in non-responders. Baseline cholesterol ester and phospholipid metabolites correlated with LDL-C response to treatment. CRP response to therapy correlated with baseline plasmalogens, lipids involved in inflammation. There was no overlap of lipids whose changes correlated with LDL-C or CRP responses to simvastatin suggesting that distinct metabolic pathways govern statin effects on these two biomarkers. Metabolic signatures could provide insights about variability in response and mechanisms of action of statins.

Keywords Cardiovascular disease $\cdot$ Lipidomics . Metabolomics · Pharmacogenomics .

Pharmacometabolomics · Simvastatin

\section{Introduction}

Statins or HMG-CoA reductase inhibitors are used to treat or prevent cardiovascular disease (CVD), which affects over 64 million Americans and remains the leading cause of death for all major ethnic groups in the U.S. (AHA 2004). Statins are the largest single class of drugs prescribed for CVD prevention, with over 120 million prescriptions and over $\$ 13$ billion in sales in 2003 (Lawrence and Zaugg 2004). Several clinical trials including Scandinavian Simvastatin Survival Study, Cholesterol and Recurrent Events, and Jupiter (Baigent et al. 2005; Ridker et al. 2008; Zhou et al. 2006) have demonstrated the beneficial effects of statin therapy for primary and secondary prevention of CVD. The primary clinical rationale for statin use is to reduce low-density lipoprotein cholesterol (LDL-C) and, thereby, to reduce CVD risk (Grundy 2001). This effect is due in large part to increased LDL and 
intermediate-density lipoprotein (Neidlinger et al. 2006) clearance as a result of the up-regulation of LDL receptors (Bilheimer et al. 1983).

Intervention trials with statin drugs have demonstrated a remarkable degree of consistency in their ability to reduce risk for both CVD and stroke (Grundy 2001; Grundy et al. 2004) nevertheless, in all of these trials, residual CVD risk remains high (60-75\%), and potential drug-related toxicity, while infrequent, is a significant concern. Response to statins varies among individuals, and mechanisms of interindividual variation in response remain poorly understood with no validated biomarkers that can adequately predict outcome. Several studies, including the cholesterol and atherosclerosis pharmacogenetics (CAP) have demonstrated a wide range of lipid and lipoprotein responses to statin therapy (Simon et al. 2006) with decrease in LDL-C for a given statin ranging from less than $5 \%$ to greater than $60 \%$, even when compliance is taken into account. Similar variation has been observed for other statin effects that can contribute to CVD outcomes, including reductions in triglyceride and very low-density lipoproteins (VLDL), increases in high-density lipoproteins (HDL), and reductions in the inflammatory marker C-reactive protein (CRP) (Sathyapalan et al. 2008; Bonnet et al. 2008; Ridker et al. 2008).

Since the correlation between statin-induced reductions in LDL-C and CRP is weak ( $r=0.13$ ) (Baigent et al. 2005), the variation in CRP response cannot be explained by LDL-C alone. Patients receiving statins have lower event rates than predicted by their achieved LDL-C, raising the possibility of distinct mechanisms beyond cholesterol reduction (cholesterol-independent effects). Some cholesterol-independent or "pleiotropic" beneficial effects of statins include: improvement of endothelial function, reduction of platelet activity, stabilization of atherosclerotic plaques, antioxidant, anti-inflammatory and immunomodulatory effects (Jasinska et al. 2007). Many of these pleiotropic effects might be mediated via inhibition of the L-mevalonic acid pathway, blocking the synthesis of isoprenoid intermediates.

Lipidomics, or global mapping of metabolites, offers a means of identifying markers that may be indicative of pathways that modulate response to statin treatment. In this study, we performed such analyses in pre- and post-treatment plasma samples from 48 individuals selected from the upper and lower tails of the distribution of LDL response to simvastatin treatment in the CAP trial. Out of 300 lipids measured within seven lipid classes, we identified lipids whose baseline concentrations predicted statin-induced changes in LDL-C and CRP, and also examined correlations of these changes with changes in the lipidomic measurements. In this manner, we were able to identify distinct lipid signatures for statin effects in subjects who do and do not respond significantly to treatment as defined by changes in on these two CVD risk markers.

\section{Materials and methods}

Samples were selected from the Cholesterol and Pharmacogenetics (CAP) Study, study, which was designed to examine genetic and non-genetic factors affecting the response to simvastatin therapy among African-Americans and whites. With response to therapy defined as the percentage change in LDL cholesterol after treatment, a group of 12 "good" responders were selected at random from the top $10 \%$ of responders in the CAP study and matched for body mass index, race, and gender to 12 subjects in the lowest $10 \%$ of responders, considered "poor" responders. The sample size was calculated for each metabolite, based on variability found in previous metabolomics studies, at a fixed significance $(\alpha=0.05)$ and power $(\beta=0.8)$. Twelve subjects per group were selected to provide $10-50$ significant metabolite differences between the groups. The initial analysis was conducted to generate hypotheses of possible differences between good and poor responders. A second set of good and poor responders was selected and analyzed separately, for a total of 24 good and 24 poor responders. Data from each set are presented as supplemental material. The subjects in the two groups were selected at random so the data could be analyzed as a combined set. Since the main findings did not differ significantly between the two subgroups, the data sets were combined to increase the sample size and power, analyzed, and the results presented in this paper.

The study population has been reported previously (Simon et al. 2006). Subjects were 944 participants, aged 60 years, with a baseline total serum cholesterol level of $160-400 \mathrm{mg} / \mathrm{dl}$. Participants were followed for a total of 6 weeks on simvastatin therapy ( $40 \mathrm{mg}$ at bedtime) and were seen at clinic visits conducted at 2-week intervals. Blood specimens from each subject were obtained after overnight fast at baseline and 6 weeks of therapy.

An assessment of plasma lipid profiles was performed, as described previously (Watkins et al. 2002). Quantitative measurements of fatty acids in lipid classes were determined as nmol fatty acid/gram of plasma. SFA, MUFA, PUFA, n3, n6, n7, and n9 fatty acid groups were calculated. Metabolites were identified by the lipid class and the fatty acid moiety, e.g. arachidonic acid in phosphatidylcholine: PC20:4n6. Abbreviations for lipid classes are as follows: cholesterol esters (CE), diacylglycerols (DG), free fatty acids (FA), lysophosphatidylcholine (LYPC), phosphatidylcholine (PC), phosphatidylethanolamine (PE) and triacylglycerols (TG), free cholesterol (FC), cholesterol (CH), polyunsaturated fatty acid (PUFA), monounsaturated fatty 
acid (MUFA), saturated fatty acid (SFA), plasmalogen (dm), total lipid class (LC), very low density lipoprotein (VLDL), low density lipoprotein (LDL), C-reactive protein (CRP).

In addition, fatty acids are identified first by the number of carbons in the molecule (e.g. 20), the number of double bonds in the molecule (e.g. 4), and lastly the position of the double bonds (e.g. n6). Thus PC20:4n6 would be a phosphatidylcholine molecule containing a 20 carbon fatty acid with 4 double bonds, at the $\mathrm{n} 6$ position.

Heat maps were created to visualize differences in metabolites pre- and post-treatment and to evaluate correlation among metabolites and drug responses. Data from the paired $t$-tests were used for pre- and post-treatment heat maps. For treatment effects significant at an $\alpha=0.05$, mean percentage difference in the concentration of the metabolite induced by treatment was calculated. The pre- and posttreatment heat maps can be read as follows: the column headers display the fatty acid and the row headers the family of fatty acids present in each lipid class. Metabolites significantly increased by treatment are displayed in red; those decreased are displayed in green. Correlation heat maps were created using the correlation analysis described below. Treatment effects significant at a $P<0.05$ were included in the heat map with blue as a positive correlation and yellow as negative. The brightness of each color corresponds to the magnitude of the difference in quartiles with the brighter the square, the larger the difference. Spearman's correlation coefficients were used to assess relationships between pretreatment metabolites and baseline LDL or CRP concentration. $q$-values were calculated for all correlation analyses (Storey and Tibshirani 2003).

To test associations of metabolites with LDL-C, we utilized Wilcoxon rank test to evaluate the differences between good and poor responders pre-treatment, posttreatment, and post-treatment minus pre-treatment, and Spearman's correlation coefficients to assess relationships between metabolites and LDL-C or CRP. Metabolite changes were defined as post-treatment minus pre-treatment level. Lipid changes were correlated with LDL-C or CRP response to treatment by calculating their Spearman's correlation coefficients with the post-treatment level of LDL or CRP, adjusting for pre-treatment level. For predicting LDL or CRP response to statin treatment, Spearman's correlation coefficients were calculated using pre-treatment metabolites with post-treatment level of LDL or CRP, adjusting for pre-treatment level.

Activities of enzymes involved in fatty acid synthesis were estimated using product to precursor ratios: 18:3(n-6)/ $18: 2(\mathrm{n}-) 6$ or $24: 5(\mathrm{n}-6) / 24: 4(\mathrm{n}-6)$ for delta 6 desaturase; $20: 3(\mathrm{n}-6) / 18: 3(\mathrm{n}-6), \quad 22: 4(\mathrm{n}-6) / 20: 4(\mathrm{n}-6)$ or $\quad 24: 4 \mathrm{n} 6 / 22: 4$ (n-6) for elongase; and 20:4(n-6)/20:3(n-6) for delta 5 desaturase. The graphic is displayed with activities or metabolites significantly increased by treatment in red and those decreased in green.

\section{Results}

With response to therapy defined as the percentage change in LDL cholesterol after treatment, 24 "good" responders were selected from the top $10 \%$ of responders in the CAP study and matched for body mass index, race, and gender to 24 subjects in the lowest $10 \%$ of responders, considered "poor" responders. Demographics for the two groups are shown in Table 1. Because this is a select subgroup of subjects, demographics vary slightly from the full study population. Good responders were slightly older than poor responders, with marginally higher initial cholesterol, HDL cholesterol, ApoA1, ApoB, and CRP levels. The 48 subjects in this analysis were older and included a lower percentage of males and African Americans than the remainder of the subjects from the top and bottom $10 \%$ of subjects in the full study (Age: $52.93 \pm 12.61,55 \%$ male, $46 \%$ African American). CRP levels were not reported in the original paper.

The effects of simvastatin treatment on concentrations of seven lipid classes in the good and poor responder groups are shown in Fig. 1. The good responders showed decreases in concentrations of CE, FC, PC, and PE while both good and poor responders decreased the amount of TG. In good responders, most of the fatty acid metabolites decreased with statin treatment, however there were specific fatty acids within the FA and LY classes that increased in concentration. In poor responders, fewer changes were seen, and five specific metabolites increased in concentration.

In addition to metabolite concentration changes, the mole percentages of all fatty acids within each lipid class were used to define compositional changes within lipid classes. This approach assigns a total class as $100 \%$ and each fatty acid within the class is calculated as $\%$ of the total lipid class, allowing evaluation of whether individual fatty acids changed disproportionately within a class. Approximately 40 metabolites were altered by drug treatment in the good responders but not in the poor responders (Supplemental data). Of metabolites that were increased selectively in the good responders, 13 of 16 contained saturated or monounsaturated fatty acids. Among metabolites that were selectively decreased in good responders, six were total lipid classes and 15 of 18 were PUFAs. There were seven metabolites with mole percentages changed similarly by treatment for both groups (Fig. 2). Four metabolites contained arachidonic acid (20:4n6) and were increased in both groups. The mole percentage of arachidonic acid increased post-treatment in all lipid classes for both good and poor responders, although not all changes 
Table 1 Demographics of patients included in metabolomics study

Values are mean $\pm \mathrm{SD}$

\begin{tabular}{llll}
\hline & Good responders & Poor responders & $P$ value \\
\hline Total $(n)$ & 24 & 24 & NA \\
Male $(\%)$ & 33 & 33 & NA \\
Body mass index $\left(\mathrm{kg} / \mathrm{m}^{2}\right)$ & $28.5 \pm 6.4$ & $29.1 \pm 5.1$ & NA \\
Race\% African American & 25 & 29 & NA \\
$\%$ decrease in LDL cholesterol & $63.1 \pm 4.2$ & $7.6 \pm 9.4$ & $<0.0001$ \\
Age (years) & $60.3 \pm 13.8$ & $53.1 \pm 12.4$ & 0.0634 \\
Initial LDL-C (mg/dl) & $133.2 \pm 31.9$ & $120.1 \pm 29.6$ & 0.1451 \\
Initial HDL-C (mg/dl) & $58.2 \pm 15.5$ & $53.7 \pm 18.6$ & 0.3651 \\
Initial ApoA1 (mg/dl) & $141 \pm 29.7$ & $127.2 \pm 27.4$ & 0.0982 \\
Initial ApoB (mg/dl) & $102.6 \pm 21.0$ & $91.7 \pm 21.1$ & 0.0791 \\
Initial total cholesterol (mg/dl) & $219.5 \pm 39.5$ & $201.8 \pm 32.7$ & 0.0957 \\
Initial CRP & $3.2 \pm 5.0$ & $1.8 \pm 1.9$ & \\
Final CRP & $2.7 \pm 4.8$ & $1.4 \pm 1.7$ & \\
\hline
\end{tabular}

reached statistical significance. Changes unique to good responders included decreased total lipid classes and numerous phospholipid metabolites, with increases in several saturated fatty acids.

Because the samples were selected to differentiate those with the best or worst LDL-C response to statins, we wanted to test which metabolites were correlated with response. For all the correlation analyses, all subjects were combined and analyzed as a single group. We first evaluated which metabolites at baseline (concentration and composition) were correlated with baseline LDL-C concentration. Baseline LDL-C showed a strong positive correlation primarily with baseline metabolite concentrations of CE and PC (Fig. 3a, $q$-values for measure of false discovery rate: $0.07-<0.0001)$. With baseline mole percentage compositional data, baseline LDL-C primarily correlated with metabolites containing n-3 fatty acids (Fig. 3a, $q$-values: $0.2-0.08)$. We next evaluated which baseline metabolites, (concentration and composition), were correlated with response to simvastatin treatment as measured by LDL-C change (Fig. 4). Baseline concentrations of specific n-6 and n-3 metabolites were positively correlated with LDL-C response to statin treatment (Fig. 4a). In the mole percentage data, CE and DG SFA were negatively and DG-n6 and FA-n3 metabolites were positively correlated with LDL-C outcome (Fig. 4b, $q$-values: 0.7 for $\mathrm{n}$ mole and 0.58 for mole percentage data).

Finally, we evaluated the changes in metabolites pre- to post-treatment that were correlated with changes in LDL-C response to treatment. Figure $3 \mathrm{~b}$ shows inverse correlations between the changes in concentration of $\mathrm{CE}, \mathrm{PC}$ and $\mathrm{PE}$ and responses of LDL-C to treatment. Larger decreases in metabolite concentrations were correlated with larger responses to treatment ( $q$-values: $0.2-<0.01)$. Correlations with changes in mole percentage identified specific fatty acid metabolites within the $\mathrm{CE}$ and PC lipid classes with positive correlations with the LDL-C response to treatment. For these metabolites an increase in the mole percentage with simvastatin treatment was associated with a greater LDL-C response to treatment ( $q$-values: 0.3-0.03).

CRP was used as a marker of inflammation and a secondary outcome in this study. There were very few baseline metabolites whose concentration correlated with baseline CRP (Fig. 5, $q$-values 0.9-0.2). When evaluating baseline composition mole percentage of lipids within major lipid classes (mole percent), we found that saturated fatty acids and 18:2n6 metabolites across several lipid classes correlated with baseline CRP concentration ( $q$-values: 0.3-0.07).

Baseline metabolite concentration and composition data were correlated with the CRP response to simvastatin treatment (Fig. 4). We found that baseline metabolite concentrations of PE plasmalogens (dm) were positively correlated with the treatment-induced changes in CRP (Fig. 4), while PC plasmalogens were negatively related to changes in CRP ( $q$-values: $0.9-0.07)$. When we evaluated mole percentage data, again we found PE plasmalogens positively correlated with CRP outcome, although $q$-values were 0.5 .

Finally, we evaluated the correlations between the changes in metabolites pre- to post-treatment and CRP response to treatment. There were very few metabolites whose concentration changes correlated with CRP response to statins (Fig. 4b, $q$-values $=0.9$ ). However, the mole percentage data showed significant correlations between changes in $\mathrm{n} 6$ and PUFA metabolites across lipid classes with CRP response to treatment ( $q$-values: $0.3-0.07$ ).

\section{Discussion}

Statins are highly effective cholesterol lowering agents. However, there is a very large inter-subject variation in the 

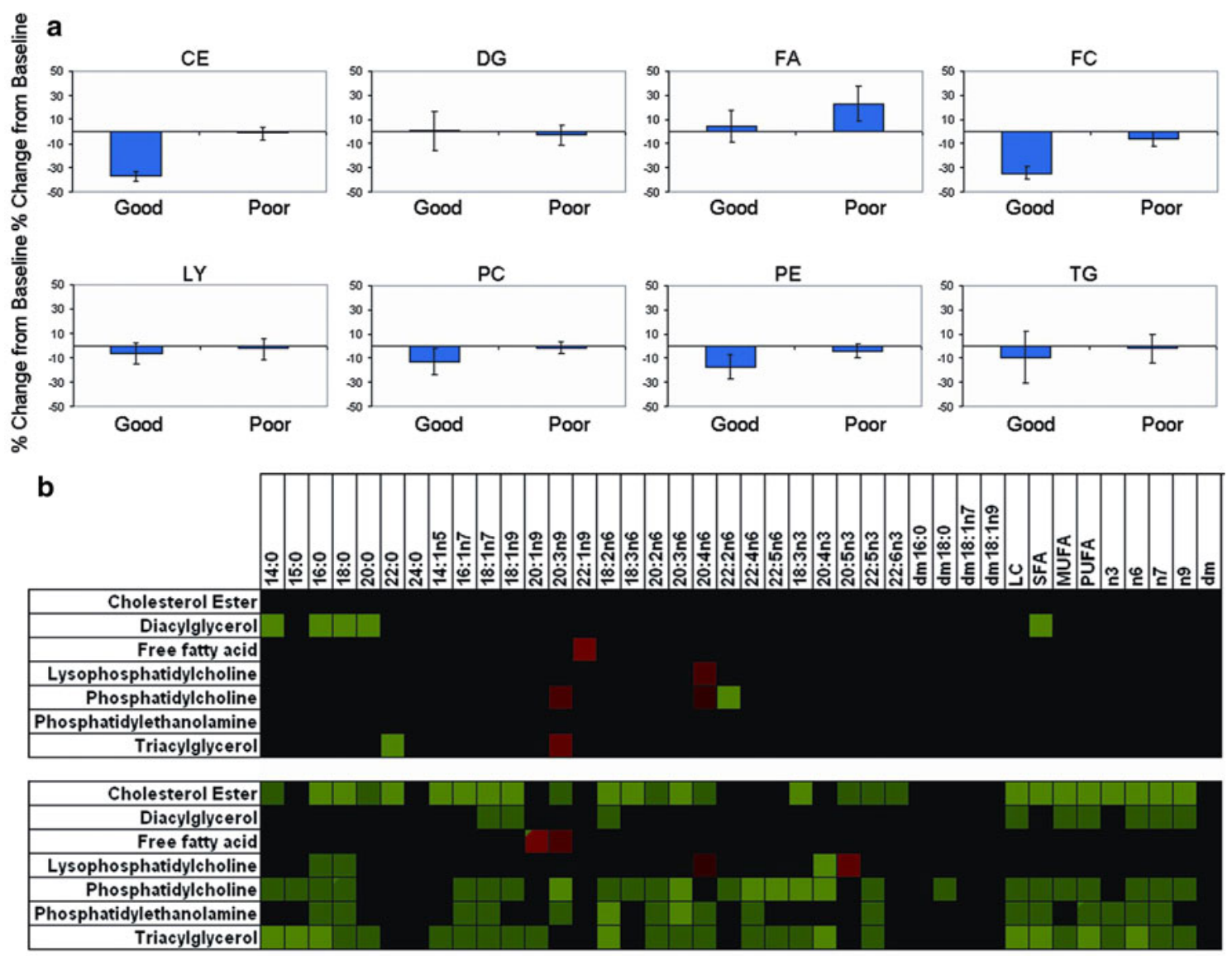

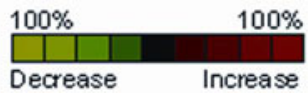

Fig. 1 Changes in effect of simvastatin treatment on lipid classes post-treatment in good and poor responders with simvastatin response defined as change in LDL-C. a Data is mean and standard deviation of the percent difference in each lipid class post-treatment. b Heat map showing quantitative changes $(\mathrm{nmol} / \mathrm{g}$ ) in lipids post-treatment with simvastatin in poor responders, (top) and in good responders (bottom). The column headers indicate fatty acid metabolites as they appear in

response to statin therapy, and the mechanisms responsible for such variability remain poorly understood. This observation holds when outcome is defined as a cardiovascular event or measured as a biomarker such as LDL-C or CRP. Moreover, single biomarkers provide limited prediction for clinical response to treatment. This study was designed to exploit the large differences in response to simvastatin treatment in the CAP trial and enable the development of metabolite signatures that predict individual response to treatment. Our primary biomarker of response to simvastatin treatment was change in LDL-C. The study subjects were selected to provide data at both extremes of the range of differences seen with treatment. In addition, we used CRP as a secondary measure of treatment response. Both LDL-C and CRP have been shown to be predictive of cardiovascular outcome in large statin trials, however, each each distinct lipid class (rows). Lipids whose percent levels were significantly $(P \leq 0.05)$ higher post- versus pre-treatment are shown in red while those with significantly $(P \leq 0.05)$ decreased level are shown in green with the brightness of each color corresponded to the magnitude of the difference in quartiles with the brighter the square, the larger the difference

biomarker has limited predictive power for individual cardiovascular outcome. Because this work was initiated as a pilot study, the experimental design was to accentuate the differences between the extremes. Additional studies are underway to investigate the compete spectrum of response from patients in the CAP trial. The CAP trial was selected for the initial and the larger study so the metabolomic data collected in these studies and the interpretation of that data could be incorporated with the pharmacogenomic data being collected as part of the original trial design.

Metabolomics applies powerful analytical tools that enable mapping of biochemical pathways modified by disease or drug treatment. In addition, metabolomic "signatures" present in patients who do and do not respond to drug therapy, i.e., signatures that reflect the drug response phenotype, could lead to mechanistic hypotheses that 
a

b

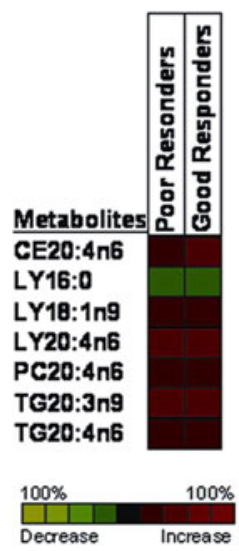

Metabolites

CE16.0

CE18.0

CE24.0

CE18:1n7

CE22:1n9

CE22:6n3

DG18:0

FA20:1n9

LY14:1n5

LY20:5n3

PE20:0

PE14:1n5

PC18:1n7

PC22:6n3

TG24:1n9

TG18:3n6

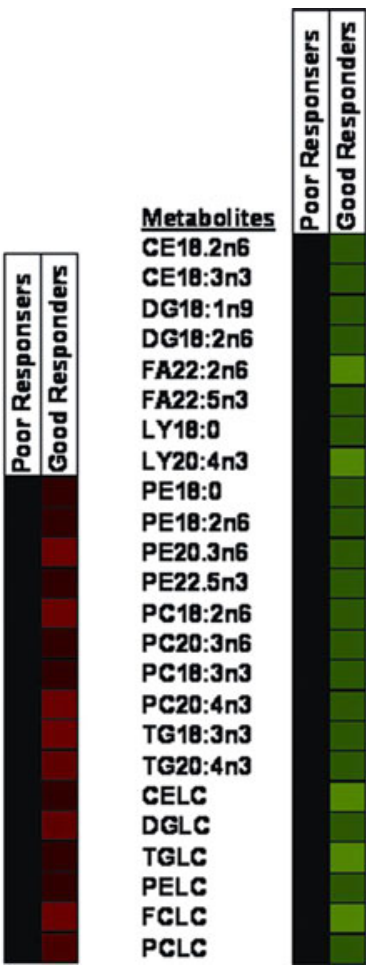

Fig. 2 Heat maps showing compositional changes within lipid classes (mole percentage changes) post-treatment with simvastatin. a Data were sorted to select metabolites that were significantly $(P \leq 0.05)$ changed pre- to post-treatment in the same direction for both good and poor responders. b Data were sorted to select metabolites that were significantly $(P \leq 0.05)$ changed pre- to posttreatment in good responders only. Metabolite changes are listed on the right. Lipids whose percent levels were higher post- versus pretreatment are shown in red while those with decreased level are shown in green; see Sect. 2 for details with the brightness of each color corresponded to the magnitude of the difference in quartiles with the brighter the square, the larger the difference

provide insights into the underlying basis for individual variation in drug response. This study defines global effects of simvastatin on metabolite concentrations and distribution within multiple lipid classes.

The largest treatment effects induced by simvastatin were decreased total lipid class changes (Fig. 1). The changes in some lipid classes have been studied previously (Simon et al. 2006; Ozerova et al. 2001), but no detailed map has been established that provides all of the concentration and composition of fatty acids within each lipid class. Because our samples were selected from individuals who were among the highest or lowest responders for changes in LDL concentration, it is not surprising that there were large changes in $\mathrm{CE}$ and $\mathrm{FC}$ in the good responders group while there were limited changes in the poor responders.

Other changes that were not expected included decreases in phospholipids PC and PE which have key roles in lipoprotein membrane structure and function. The concentration of $\mathrm{PC}$, in particular, was closely correlated with the concentration of $\mathrm{CE}\left(R^{2}=0.479\right.$, data not shown). We found the relationship between PC and CE concentrations held for both good and poor responders and pre- to post-treatment. We would expect this result if the changes in PC and PE concentration were in response to therapy-induced changes in cholesterol concentration. While simvastatin has been postulated to directly decrease the synthesis of phospholipids (Yanagita et al. 1994), the close relationship between CE and PC indicates potential a secondary effect of cholesterol synthesis on phospholipids rather than a direct effect of simvastatin.

Precursor/product ratios were used to estimate changes in the production of specific metabolites. Good responders had a significant increase in the ratio of $20: 4 n 6 / 20: 3 n 6$. This ratio was used as a measure of delta- 5 desaturase activity. Simvastatin treatment has been shown to increase the activity of delta- 6 and delta- 5 desaturases via SREBP resulting in increased formation of arachidonic acid from linoleic acid (18:2n6) (Rise et al. 2007). In this study, only good responders had a statistically significant increase in delta-5 desaturase product/substrate ratios (20:4n6/20:3n6), yet both the good and the poor responders had significant increases in the mole percentage of arachidonic acid within multiple lipid classes (Figs. 2, 6). In addition, the good responders had decreased mole percentages of 18:2n6 across multiple lipid classes, again emphasizing the increased activation of the metabolic pathway. Previous research has shown the decreased linoleic acid and increased arachidonic acid could be the result of increased delta-5 and delta- 6 desaturase activities in response to simvastatin treatment (Jula et al. 2005; Rise et al. 2007; Rise et al. 2001). However, the previous published research did not differentiate either between lipid classes or between good and poor responders. Good responders appeared to have had a much stronger increase in the use of linoleic acid for the production of arachidonic acid than the poor responders, and this effect was detected in both PC and TG (Fig. 6).

Interestingly, PC18:3n3 and TG18:3n3 were also decreased in good responders with statin treatment. The same hepatic enzymes that modify $\mathrm{n} 6$ fatty acids will also desaturate and elongate 18:3n3-22:5n3 and 22:6n3. In the good responders, we did find a significant increase in the percentage of PC22:6n3, but not TG22:6n3, perhaps reflecting the lower concentration and percentage of this metabolite in TG. This study demonstrates that the changes in desaturation and elongation of PUFAs were more pronounced in good responders, but were also present in the poor responders (Figs. 2, 5).

Differences in the composition of CE, specifically CE palmitic (16:0) and arachidonic acid and the ratio of 16:0/ 20:4n6, have been associated with atherosclerotic potential 
a

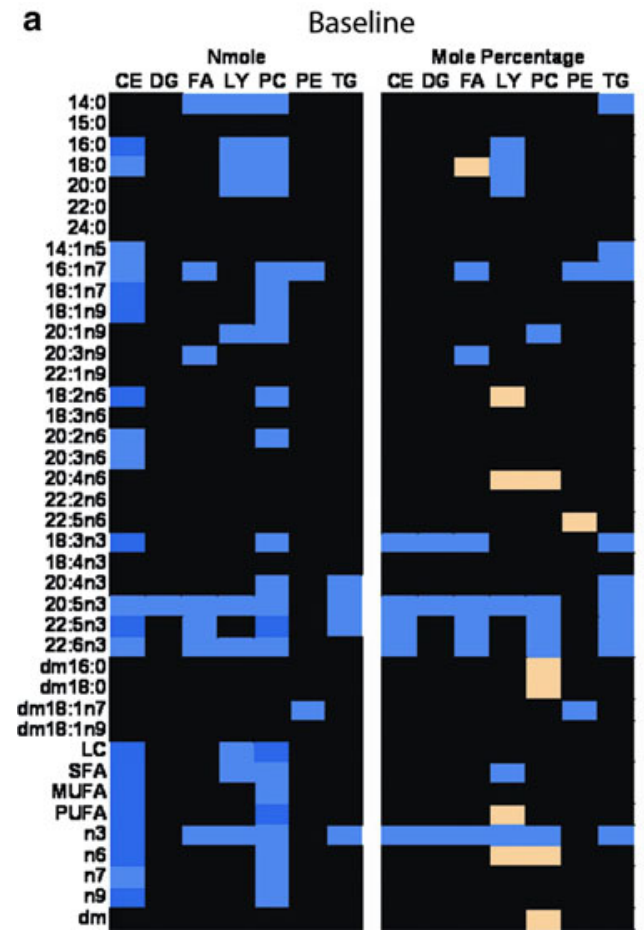

Fig. 3 Correlation between LDL-C and metabolite concentration and composition. Heatmaps show significant $(P \leq 0.05)$ metabolite correlations with LDL-C concentration at baseline and post-treatment with simvastatin treatment. Fatty acids are listed on the left side of each heat map with lipid classes listed across the top. Each square represents a correlation between the metabolite and LDL-C. a Baseline metabolite concentrations (nmol/g, left side) or mole percentages (right side) correlated with baseline LDL-C concentration, b Change

and risk of coronary disease (Liu et al. 1995; Messner et al. 1998; Sundstrom et al. 2001; Ma et al. 1997; Lee et al. 2004). Studies have indicated a relationship between CE composition and insulin resistance or metabolic syndrome, known risk factors for CVD (Moilanen et al. 1986; KleinPlatat et al. 2005). In this study, the ratio of CE16:0/20:4n6 was similar in both good and poor responders pre-treatment (1.57 \pm 0.42 and $1.52 \pm 0.28$, respectively), and changed significantly post-treatment, but the ratio decreased more in the good responder group $(\operatorname{good} 1.22 \pm 0.27(P<0.0001)$ and poor $1.38 \pm 0.29(P=0.0066)$ responders $)$.

We used correlation analysis to evaluate which baseline metabolites were related to pre-treatment LDL-C concentrations and which changes in metabolites were related to LDL-C response to statins. Strong correlations with CE and PC metabolites were found for both the baseline correlations and the change in concentration correlations. These changes confirm our observation that the amount of lipoprotein cholesterol at baseline influences the amount of response to simvastatin. Of interest is the observation that the baseline mole percentages of EPA (20:5n3) and DHA (22:6n3) are positively correlated with the baseline concentrations of

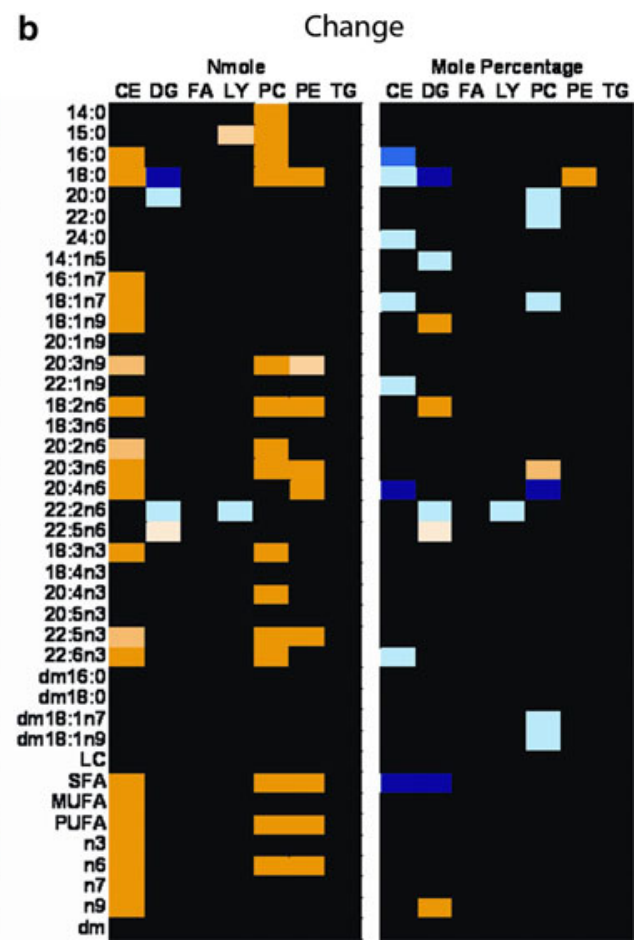

in metabolite concentrations ( $\mathrm{nmol} / \mathrm{g}$, left side) or lipid composition (mole percentage changes right side) pre- to post-treatment correlated with LDL-C response to treatment. Blue is a positive correlation and yellow is a negative correlation. The darkness of each color corresponds to the magnitude of the difference in quartiles of the Spearman correlation coefficient with the darker the square, the larger the difference

LDL-C. This may reflect a dietary effect on cholesterol concentrations.

Simvastatin treatment may reduce inflammation independent of its effects on cholesterol biosynthesis (Sotiriou and Cheng 2000). Changes in cytokine biomarkers of inflammation and eicosanoid products have been identified with statin treatment (Cipollone et al. 2003). As statin treatment has anti-inflammatory effects, the rise in the proinflammatory substrate, arachidonic acid, in many lipid classes is interesting. This may reflect a decreased use of this fatty acid for synthesis of inflammatory lipids. Since the increased compositional changes in arachidonic acid were found in both the good and poor responders, if this effect is real, it is likely that the anti-inflammatory effects of simvastatin occur in both groups. CRP was measured in the CAP study, but the results were not presented in the original paper. We used CRP as a marker of inflammation in this study, hypothesizing that both groups would have differences in inflammation and that the inflammation-induced changes in lipids would be independent of LDL-C-related changes. The absolute magnitude of the change in CRP was of similar magnitude for 


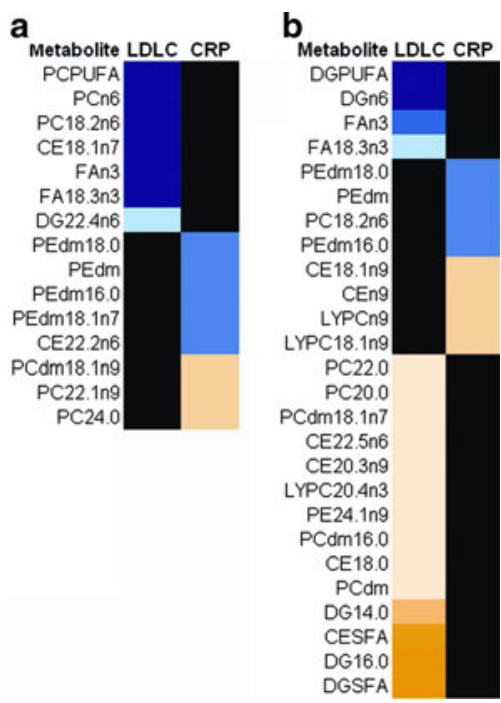

Fig. 4 Baseline metabolites which correlate with LDL-C or CRP response to treatment. Sorted heat maps showing metabolite concentration $(\mathrm{nmol} / \mathrm{g}$ ) (a) or (b) lipid composition within classes (mole percentage) $\mathbf{b}$ that are predictive of changes in LDL-C or CRP $(P \leq 0.05)$. Metabolites are listed on the left side of each sorted heat map. Each square represents a correlation between the metabolite and LDL-C or CRP. Blue is positive and yellow is negative correlation. The darkness of each color corresponds to the magnitude of the difference in quartiles of the Spearman correlation coefficient with the darker the square, the larger the difference

both groups (Table 1); however the relative percentage change was higher in the poor responders than the good responders (22\% vs. 15\%, respectively). Because these subjects were selected based their change in LDL-C with statin treatment, the change in CRP is biased towards good or poor LDL-C response.

Correlations between metabolites and CRP were used to evaluate the relationships between the metabolites and inflammation. We found a clear positive relationship between the mole percentage of 16:0 in several lipid classes and CRP, and a negative relationship between linoleic acid and CRP at baseline. Saturated fatty acids have been associated with increased inflammation and increased linoleic acid is known to decrease inflammatory markers (Ferrucci et al. 2006). This analysis thus confirms the known relationships between lipid metabolism and inflammation in our subjects. For each of the correlation analyses, there was virtually no overlap in the metabolites correlated with CRP and those correlated with LDL-C, consistent with the finding that the change in CRP was independent of the change in LDL-C.

Finally, we sought baseline metabolites that predicted the outcome of statin treatment, either LDL-C or CRP. Metabolites whose concentration was most predictive of LDL changes were PC18:2n6 and related PC lipid families, CE18:1n7, and FA18:3n3 and the related FAn3. The PC and $\mathrm{CE}$ metabolites were also correlated with baseline LDL concentrations. Since baseline LDL concentrations were also predictive of response, we assume that these metabolites are indicative of the initial status of the subjects rather than being purely predictive of the change in LDL. For the metabolites whose mole percentage was predictive of LDL changes, the strongest correlations both positive and negative were found in the DGs. DGs were not predictive of baseline LDL and therefore may represent a more selective marker to predict the response to statin treatment. The DG may reflect the overall amount of lipase activity in the system and the uptake and release of lipids by peripheral tissues.

Of the metabolites whose concentration was most predictive of CRP changes, five were plasmalogens, and the others were PC and CE metabolites. Plasmalogen and PC metabolites were also most strongly predictive of CRP response when expressed in terms of lipid composition (mole percentage). Plasmalogens are ether-linked phospholipids characterized by a vinyl aldehyde at the first position of the glycerol backbone (Lessig and Fuchs 2009), and an ester-linked fatty acid in the second position. Plasmalogens function as reservoirs for second messenger fatty acids such as arachidonic acid and DHA, and have been postulated to act as antioxidants (Engelmann et al. 1994; Hahnel et al. 1999; Lessig and Fuchs 2009). Plasmalogen concentrations decrease with age and have been identified with inflammatory-related diseases such as atherosclerosis and Alzheimer's disease (Goodenowe et al. 2007; Farooqui et al. 2006; Lessig and Fuchs 2009). Baseline PE plasmalogen concentrations were positively correlated with the response to simvastatin treatment, i.e. the higher the concentration of plasmalogens at baseline, the greater reduction in CRP with simvastatin treatment. Concurrently, PC plasmalogens were negatively correlated with CRP response to treatment. Since baseline plasmalogen concentrations were not correlated with baseline CRP concentrations, we assume that we are not seeing the relationship between baseline inflammation and response to treatment, rather that plasmalogens are predictive of the ability of the system to decrease inflammation in response to statin treatment. Plasmalogens as a class, (concentrations or mole percentages), did not respond to treatment differently between good and poor responders nor did CRP. The changes in CRP and perhaps other inflammatory molecules in these subjects appear to be independent of most metabolites, but may be related to changes in both arachidonic acid and plasmalogen concentrations. From these preliminary studies, we are unable to tell whether this antiinflammatory effect would be enough to reduce the risk of CVD in the poor responders. The results of this study emphasize the need to analyze the full range of treatment responses rather than the mean effect of treatment across all subjects. Additional studies to investigate the effects of 
a

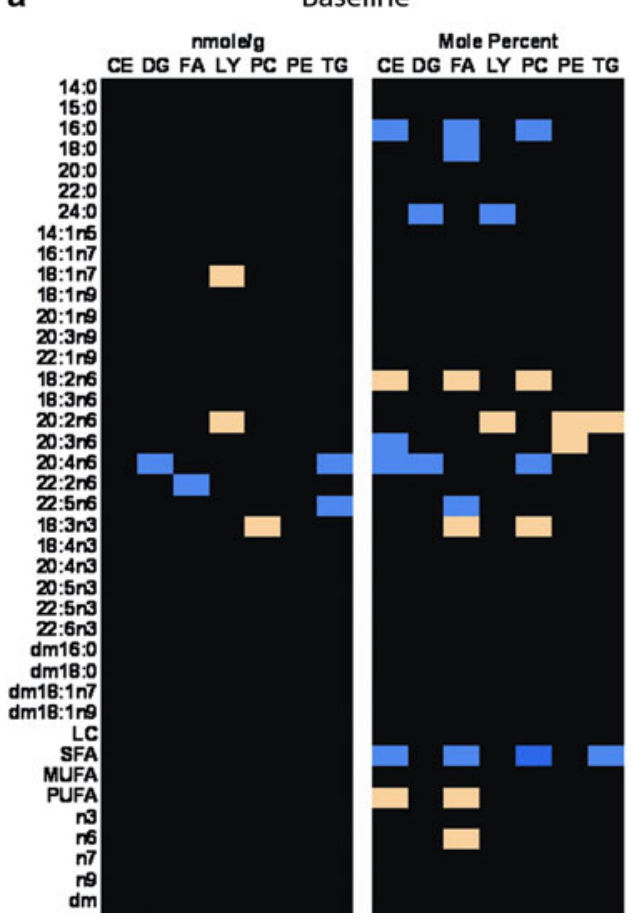

b

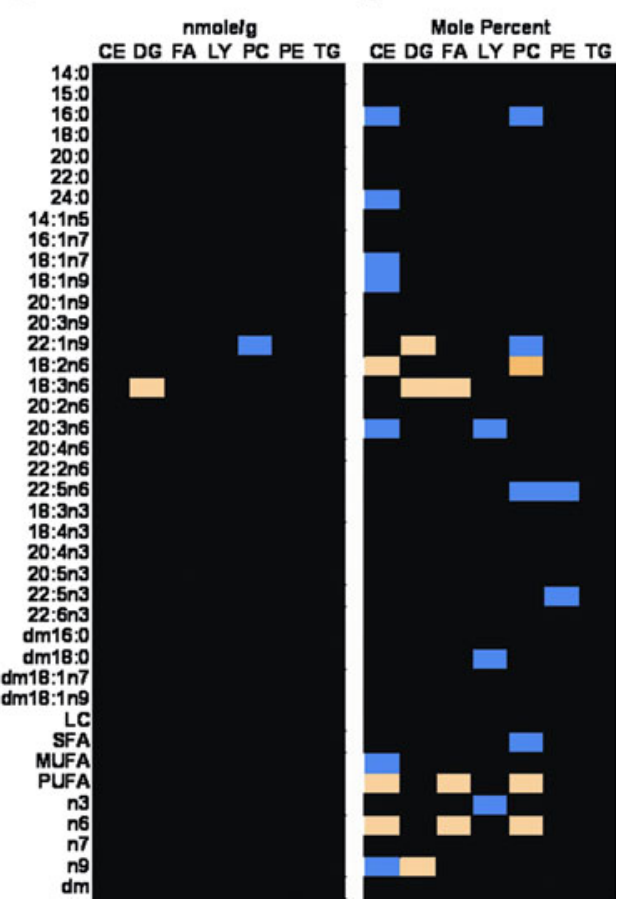

Fig. 5 Correlations between CRP and metabolite concentration and composition. Heat maps show significant $(P \leq 0.05)$ metabolite correlations with CRP concentration. Fatty acids are listed on the left side of each heat map with lipid classes listed across the top. Each square represents a correlation between the metabolite and CRP. a Baseline metabolite concentrations ( $\mathrm{nmol} / \mathrm{g}$, left side) or mole percentages (right side) correlated with baseline CRP, b Change in
Triglyceride

Good Responders Poor Responders

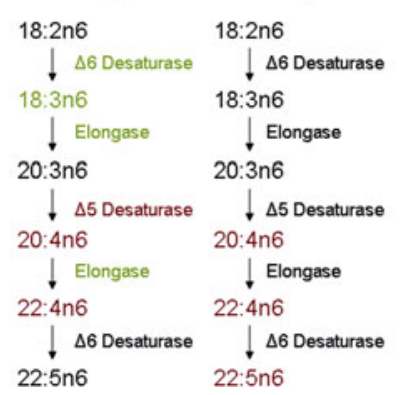

Phosphatidylcholine

Good Responders Poor Responders

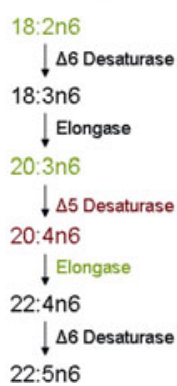

$18: 2 \mathrm{n} 6$

$\checkmark 6$ Desaturase

$18: 3 n 6$

Elongase

$20: 3 n 6$

$\checkmark \Delta 5$ Desaturase 20:4n6

Elongase

22:4n6

$\triangle 6$ Desaturase $22: 5 n 6$
Fig. 6 Metabolic pathway changes with simvastatin treatment in subjects segregated by response to LDL-C. Ratios of precursor/ products in TG and PC lipid metabolites were used to estimate changes in the metabolic pathways for biosynthesis of n6 fatty acids pre- to post-treatment with simvastatin in good and poor responders. The enzyme activities were estimated utilizing the product/precursor ratio as described in the Sect. 2 . Ratios which were significantly $(P \leq 0.05)$ higher post- versus pre-treatment are shown in red while those significantly $(P \leq 0.05)$ decreased are shown in green

simvastatin treatment on concentrations of the inflammatory lipids and cytokines in good and poor responders are underway. metabolite concentrations (nmol/g, left side) or mole percentages (right side) pre- to post-treatment correlated with CRP response to treatment. Blue is a positive correlation and yellow is a negative correlation. The darkness of each color corresponds to the magnitude of the difference in quartiles of the Spearman correlation coefficient with the darker the square, the larger the difference

The limitations of this study include the relatively small sample size, the likelihood of type 1 errors due to multiple testing although we have corrected to the degree possible by use of q values, use of a single statin and a single dose, and the possibility, as suggested in a recent report (Laaksonen et al. 2006) that metabolomic profiles can differ among individual statin drugs. Hence the present findings require confirmation in larger studies employing other statins. The major changes in the lipid profiles identified in this study, such as the total lipid classes and cholesterol changes, as well as changes in inflammation related pathways, could be shared among different members of the statin family of drugs. This is currently being investigated in larger studies and where simvastatin is compared to pravastatin.

\section{Concluding remarks}

The findings of this study point to the potential utility of metabolomic surveys for identifying downstream effects of HMG CoA reductase inhibition as well as predictors of clinical response that may have implications for assessing 
both efficacy and toxicity of this widely use class of drugs. This integration of metabolomics, in this case lipid profiling, into pharmacogenomic research can significantly enrich the information derived from the experiment and provide further avenues of research. The biochemical maps that reflect total effects of statins if correlated with clinical outcome in cardiovascular disease trials could provide novel insights into the therapeutic effects of this important class of drug.

Acknowledgments Work is supported by NIGMS grants R24 GM078233, "The Metabolomics Research Network for Drug Response Phenotype" (R.K.D., R.M.K.); and U01 HL069757, "Pharmacogenomics and Risk of Cardiovascular Disease" (R.M.K.).

Open Access This article is distributed under the terms of the Creative Commons Attribution Noncommercial License which permits any noncommercial use, distribution, and reproduction in any medium, provided the original author(s) and source are credited.

\section{References}

AHA. (2004). Heart disease and stroke statistics-2004 update. Dalls, TX: American Heart Association.

Baigent, C., Keech, A., Kearney, P. M., Blackwell, L., Buck, G., Pollicino, C., et al. (2005). Efficacy and safety of cholesterollowering treatment: Prospective meta-analysis of data from 90, 056 participants in 14 randomised trials of statins. Lancet, 366, 1267-1278.

Bilheimer, D. W., Grundy, S. M., Brown, M. S., \& Goldstein, J. L. (1983). Mevinolin stimulates receptor-mediated clearance of low density lipoprotein from plasma in familial hypercholesterolemia heterozygotes. Transactions of the Association of American Physicians, 96, 1-9.

Bonnet, J., Mcpherson, R., Tedgui, A., Simoneau, D., Nozza, A., Martineau, P., et al. (2008). Comparative effects of 10-mg versus 80-mg Atorvastatin on high-sensitivity C-reactive protein in patients with stable coronary artery disease: Results of the CAP (comparative atorvastatin pleiotropic effects) study. Clinical Therapeutics, 30, 2298-2313.

Cipollone, F., Fazia, M., Iezzi, A., Zucchelli, M., Pini, B., De Cesare, D., et al. (2003). Suppression of the functionally coupled cyclooxygenase-2/prostaglandin E synthase as a basis of simvastatin-dependent plaque stabilization in humans. Circulation, 107, 1479-1485.

Engelmann, B., Brautigam, C., \& Thiery, J. (1994). Plasmalogen phospholipids as potential protectors against lipid peroxidation of low density lipoproteins. Biochemical and Biophysical Research Communications, 204, 1235-1242.

Farooqui, A. A., Ong, W. Y., \& Horrocks, L. A. (2006). Inhibitors of brain phospholipase A2 activity: Their neuropharmacological effects and therapeutic importance for the treatment of neurologic disorders. Pharmacological Reviews, 58, 591-620.

Ferrucci, L., Cherubini, A., Bandinelli, S., Bartali, B., Corsi, A., Lauretani, F., et al. (2006). Relationship of plasma polyunsaturated fatty acids to circulating inflammatory markers. Journal of Clinical Endocrinology and Metabolism, 91, 439-446.

Goodenowe, D. B., Cook, L. L., Liu, J., Lu, Y., Jayasinghe, D. A., Ahiahonu, P. W., et al. (2007). Peripheral ethanolamine plasmalogen deficiency: A logical causative factor in
Alzheimer's disease and dementia. Journal of Lipid Research, $48,2485-2498$.

Grundy, S. M. (2001). United States cholesterol guidelines 2001: Expanded scope of intensive low-density lipoprotein-lowering therapy. American Journal of Cardiology, 88, 23J-27J.

Grundy, S. M., Cleeman, J. I., Merz, C. N., Brewer, H. B., Jr, Clark, L. T., Hunninghake, D. B., et al. (2004). Implications of recent clinical trials for the national cholesterol education program adult treatment panel III guidelines. Circulation, 110, 227-239.

Hahnel, D., Thiery, J., Brosche, T., \& Engelmann, B. (1999). Role of plasmalogens in the enhanced resistance of LDL to copperinduced oxidation after LDL apheresis. Arteriosclerosis, Thrombosis, and Vascular Biology, 19, 2431-2438.

Jasinska, M., Owczarek, J., \& Orszulak-Michalak, D. (2007). Statins: A new insight into their mechanisms of action and consequent pleiotropic effects. Pharmacological Reports, 59, 483-499.

Jula, A., Marniemi, J., Ronnemaa, T., Virtanen, A., \& Huupponen, R. (2005). Effects of diet and simvastatin on fatty acid composition in hypercholesterolemic men: A randomized controlled trial. Arteriosclerosis, Thrombosis, and Vascular Biology, 25, 1952 1959.

Klein-Platat, C., Drai, J., Oujaa, M., Schlienger, J. L., \& Simon, C. (2005). Plasma fatty acid composition is associated with the metabolic syndrome and low-grade inflammation in overweight adolescents. American Journal of Clinical Nutrition, 82, 11781184.

Laaksonen, R., Katajamaa, M., Paiva, H., Sysi-Aho, M., Saarinen, L., Junni, P., et al. (2006). A systems biology strategy reveals biological pathways and plasma biomarker candidates for potentially toxic statin-induced changes in muscle. PLoS ONE, 1, e97.

Lawrence, M. B., \& Zaugg, A. M. (2004). IMS health report. MM\&M.

Lee, R. G., Kelley, K. L., Sawyer, J. K., Farese, R. V., Jr, Parks, J. S., \& Rudel, L. L. (2004). Plasma cholesteryl esters provided by lecithin:cholesterol acyltransferase and acyl-coenzyme a: Cholesterol acyltransferase 2 have opposite atherosclerotic potential. Circulation Research, 95, 998-1004.

Lessig, J., \& Fuchs, B. (2009). Plasmalogens in biological systems: Their role in oxidative processes in biological membranes, their contribution to pathological processes and aging and plasmalogen analysis. Current Medicinal Chemistry, 16, 2021-2041.

Liu, M., Bagdade, J. D., \& Subbaiah, P. V. (1995). Specificity of lecithin:cholesterol acyltransferase and atherogenic risk: Comparative studies on the plasma composition and in vitro synthesis of cholesteryl esters in 14 vertebrate species. Journal of Lipid Research, 36, 1813-1824.

Ma, J., Folsom, A. R., Lewis, L., \& Eckfeldt, J. H. (1997). Relation of plasma phospholipid and cholesterol ester fatty acid composition to carotid artery intima-media thickness: The atherosclerosis risk in communities (ARIC) study. American Journal of Clinical Nutrition, 65, 551-559.

Messner, T., Sihm, H., \& Vessby, B. (1998). Fatty acid composition in serum among males 4-16 years after myocardial infarction. International Journal of Circumpolar Health, 57, 22-31.

Moilanen, T., Solakivi-Jaakkola, T., Viikari, J., Rasanen, L., Akerblom, H. K., Uhari, M., et al. (1986). Fatty acid composition of serum cholesteryl esters in relation to serum lipids and apolipoproteins in 3-18-year-old Finnish children and adolescents. Atherosclerosis, 59, 113-119.

Neidlinger, N. A., Larkin, S. K., Bhagat, A., Victorino, G. P., \& Kuypers, F. A. (2006). Hydrolysis of phosphatidylserineexposing red blood cells by secretory phospholipase A2 generates lysophosphatidic acid and results in vascular dysfunction. The Journal of Biological Chemistry, 281, 775-781.

Ozerova, I. N., Paramonova, I. V., Olfer'ev, A. M., Akhmedzhanov, N. M., Aleksandrova, M. A., \& Perova, N. V. (2001). Effects of 
simvastatin on the phospholipid composition of high-density lipoproteins in patients with hypercholesterolemia. Bulletin of Experimental Biology and Medicine, 132, 763-765.

Ridker, P. M., Danielson, E., Fonseca, F. A., Genest, J., Gotto, A. M., Jr, Kastelein, J. J., et al. (2008). Rosuvastatin to prevent vascular events in men and women with elevated C-reactive protein. New England Journal of Medicine, 359, 2195-2207.

Rise, P., Ghezzi, S., Carissimi, R., Mastromauro, F., Petroni, A., \& Galli, C. (2007). Delta5 desaturase mRNA levels are increased by simvastatin via SREBP-1 at early stages, not via PPARalpha, in THP-1 cells. European Journal of Pharmacology, 571, 97-105.

Rise, P., Pazzucconi, F., Sirtori, C. R., \& Galli, C. (2001). Statins enhance arachidonic acid synthesis in hypercholesterolemic patients. Nutrition, Metabolism \& Cardiovascular, 11, 88-94.

Sathyapalan, T., Atkin, S. L., \& Kilpatrick, E. S. (2008). Variability of lipids in patients with type 2 diabetes taking statin treatment: Implications for target setting. Diabetic Medicine, 25, 909-915.

Simon, J. A., Lin, F., Hulley, S. B., Blanche, P. J., Waters, D., Shiboski, S., et al. (2006). Phenotypic predictors of response to simvastatin therapy among African-Americans and Caucasians: The cholesterol and pharmacogenetics (CAP) Study. American Journal of Cardiology, 97, 843-850.
Sotiriou, C. G., \& Cheng, J. W. (2000). Beneficial effects of statins in coronary artery disease-beyond lowering cholesterol. Annals of Pharmacotherapy, 34, 1432-1439.

Storey, J. D., \& Tibshirani, R. (2003). Statistical significance for genomewide studies. Proceedings of the National Academy of Sciences of the United States of America, 100, 9440-9445.

Sundstrom, J., Lind, L., Vessby, B., Andren, B., Aro, A., \& Lithell, H. (2001). Dyslipidemia and an unfavorable fatty acid profile predict left ventricular hypertrophy 20 years later. Circulation, 103, 836-841.

Watkins, S. M., Reifsnyder, P. R., Pan, H. J., German, J. B., \& Leiter, E. H. (2002). Lipid metabolome-wide effects of the PPARgamma agonist rosiglitazone. Journal of Lipid Research, 43, 1809-1817.

Yanagita, T., Yamamoto, K., Ishida, S., Sonda, K., Morito, F., Saku, K., et al. (1994). Effects of simvastatin, a cholesterol synthesis inhibitor, on phosphatidylcholine synthesis in HepG2 cells. Clinical Therapeutics, 16, 200-208.

Zhou, Z., Rahme, E., \& Pilote, L. (2006). Are statins created equal? Evidence from randomized trials of pravastatin, simvastatin, and atorvastatin for cardiovascular disease prevention. American Heart Journal, 151, 273-281. 